APUNTE

\title{
Un Meta Análisis para Estimar el Papel de Polinizadores Nativos en la Sustentabilidad de Ecosistemas Forestales Naturales
}

\author{
González, Jorge ${ }^{1 *}$; Molina, María Paz ${ }^{1}$ e Ipinza, Roberto ${ }^{1}$ \\ ${ }^{1}$ Investigadores Instituto Forestal. Sede Bio Bio, Concepción. jgonzalez@infor.cl y mmolina@infor.cl \\ ${ }^{2}$ Investigador Instituto Forestal. Sede Los Ríos, Valdivia. robertoipinza@infor.cl \\ *Autor para correspondencia
}

\begin{abstract}
RESUMEN
Los ecosistemas forestales constituyen una de las mayores reservas de biodiversidad en el mundo. Comprender la compleja red de interacciones ecológicas que ocurren entre los componentes bióticos dentro de estos ecosistemas, y cómo las fuerzas de evolución dirigen estas interacciones, siempre ha sido un campo desafiante. No obstante, es importante dar este salto conceptual, debido a que las especies no evolucionan en el vacío, más bien son el producto de las interacciones de cientos de especies que coexisten en entornos variables.

El presente trabajo tiene como objetivo aportar antecedentes preliminares sobre interacciones, tomando como marco de estudio las comunidades de especies arbóreas y polinizadores nativos y exóticos. Para esto se recopilan, interacciones reportadas en la literatura. Irrefutablemente la degradación de los bosques está alterando las redes ecológicas, como las interacciones planta-polinizador, entre otras. Sin embargo, el conocimiento de los posibles efectos que subyacen a la pérdida de especies arbóreas sobre estas interacciones es aún limitado, y no es posible cuantificarlo. Se enfatiza que este trabajo es preliminar y no tiene como objetivo compilar para discutir las interacciones reportadas en este marco de estudio, pero sí se espera incentivar a la comunidad científica a profundizar en esta materia con nuevos antecedentes teóricos y desarrollo de pruebas experimentales, a través de una investigación multidisciplinaria.
\end{abstract}

Palabras claves: Ecosistemas forestales, Interacciones ecológicas, Polinizadores.

\begin{abstract}
SUMMARY
Forest ecosystems constitute one of the largest reserves of biodiversity in the world. Understanding the complex net of ecological interactions that occur between biotic components within these ecosystems, and how the forces of evolution direct these interactions, has always been a challenging field. However, it is important to make this conceptual leap, because species do not evolve in a hole, rather they are the product of the interactions of hundreds of species that coexist in variable environments.

The present work aims to provide preliminary information on interactions, taking as a framework of study the communities of tree species and native and exotic pollinators. For this, interactions reported in the literature are collected. Irrefutably, forest degradation is altering ecological networks, such as plant-pollinator interactions, among others. However, the knowledge of the possible effects that underlie the loss of tree species on these interactions is still limited, and it is not possible to quantify it. It is emphasized that this work is preliminary and does not aim to compile to discuss the interactions reported in this study framework, but it is hoped to encourage the scientific community to delve into this matter with new theoretical background and development of experimental tests, through of a multidisciplinary investigation.
\end{abstract}

Keywords: Forest ecosystems, Ecological interactions, Pollinators.

\section{INTRODUCCIÓN}

Los ecosistemas forestales constituyen una de las mayores reservas de biodiversidad en el mundo, desde los bosques boreales del extremo norte pasando por las pluviselvas tropicales hasta los bosques templados del sur. En conjunto, contienen más de 60.000 especies arbóreas diferentes y proporcionan hábitats para el $80 \%$ de las especies de anfibios, el $75 \%$ de las especies de aves y el $68 \%$ de las especies de mamíferos. Alrededor del $60 \%$ de todas las plantas vasculares se encuentran en los bosques tropicales (FAO - PNUMA, 2020).

Por consiguiente, la conservación de los ecosistemas forestales no solo permite el aseguramiento de los productos y beneficios ecosistémicos que estos brindan a la sociedad, sino que paralelamente permite la 
conservación de la gran cantidad de especies que constituyen las diferentes comunidades que estos ecosistemas albergan.

Lamentablemente, después de un exhaustivo meta análisis Medel et al. (2017) identificó 69 artículos publicados sobre 235 especies de plantas nativas de 62 familias, comenta que se ha estudiado menos del 7,9\% de las especies con flores que habitan la zona mediterránea chilena y que la mayoría de los estudios se restringió a una sola localidad y una temporada reproductiva. Además, dichos autores establecen que para aumentar la comprensión del papel de las relaciones planta-polinizador para el mantenimiento de la biodiversidad se debería ampliar el alcance taxonómico y geográfico, aumentar las escalas espacial y temporal, y aumentar el número de estudios sobre redes de polinización, ya que proporcionan estimaciones de la complejidad de la comunidad y estabilidad putativa, estimando la importancia de la polinización para los parámetros demográficos y la conservación de las plantas, y realizando estudios que estimen cuantitativamente el servicio ecológico proporcionado por los polinizadores nativos chilenos para la agricultura y el rendimiento de cultivos sostenibles.

Comprender la compleja red de interacciones ecológicas que ocurre entre los componentes bióticos a diferentes niveles (población, comunidad y/o paisaje) y cómo las fuerzas de evolución dirigen estas interacciones (Benfey y Mitchell-Olds, 2008), siempre ha sido un campo desafiante para los especialistas. No obstante, es importante dar este salto conceptual, debido a que las especies no evolucionan en el vacío. Más bien, son el producto de las interacciones de cientos de especies que coexisten en entornos variables. Entender esta mirada requiere ubicar a la comunidad y el ecosistema dentro de un marco evolutivo definido por las interacciones ecológicas y genéticas que existen entre las especies.

La Genética de Comunidades, término introducido por Antonovic (1992). como un moderno intento de integrar la ecología de comunidades y la genética cuantitativa para comprender los procesos de evolución a niveles superiores y cuyo fundamento, tanto teórico como aplicado, sostiene que conocer cómo se organizan las comunidades es fundamental para comprender los principios de ensamble y evolución de los ecosistemas, y permite utilizar las comunidades en un marco evolutivo definido por las interacciones genéticas y ecológicas que existen entre las especies que la componen (Whitham et al., 2003; 2006; 2020).

La aplicación de la genética de comunidades en ecosistemas forestales puede ser una gran ventaja para generar planes de acción para el uso y la conservación de estos recursos. Las siguientes peguntas podrían ser respondidas a través de estudios teóricos y empíricos: ¿Cuáles son las interacciones que dirigen la evolución de las especies forestales? ¿Sí la pérdida de diversidad de especies forestales altera las interacciones funcionales de la comunidad a la que pertenece, cuánta es la pérdida de las especies interactuantes? ¿La diversidad genética de especies arbóreas aporta a la estructuración de la comunidad de polinizadores? ¿En qué medida los polinizadores han seleccionado los rasgos florales en especies forestales?

Los árboles constituyen un conjunto de oferentes de polen, néctar y refugio, a un gran conjunto de comunidades de insectos y mamíferos polinizadores. A su vez, los árboles se ven beneficiados por la polinización que realizan las comunidades de insectos, quienes actúan cómo vectores, participando activamente en el flujo genético de la especie, lo que permite mantener su diversidad genética.

Sin diversidad genética, una especie no puede evolucionar y adaptarse a los cambios ambientales, experimentando una posibilidad mayor de extinción (Templeton, 1994; Donoso y Gallo, 2004). Las especies tienen tres opciones que les permiten sobrevivir ante los rápidos cambios ambientales: i) Dispersión, es decir una migración desde los hábitas nativos de las especies a otros hábitats más favorables, ii) Plasticidad fenotípica, la cual se refiere a la capacidad de un organismo de producir fenotipos diferentes en respuesta a cambios en el ambiente (no considera cambio genético) y iii) Adaptación, que se refiere al uso de la diversidad genética de las especies, para explorar variantes genéticas más adecuadas, en términos de eficiencia biológica (Stadler y Stephens, 2002; Jump et al., 2009)

Dada la importancia económica y ecológica de la polinización. La realización de estudios teóricos y experimentales que diluciden las interacciones entre especies forestales nativas e insectos polinizadores nativos y exóticos resulta un escenario muy prometedor, y cuyos resultados contribuirían evidentemente a la generación de planes de acción más integradores y de largo plazo. 
Conscientes de las limitaciones y tareas pendientes establecidas por Medel et al. (2017), en el ámbito de la investigación de los polinizadores chilenos, el presente trabajo tiene como finalidad aportar antecedentes preliminares para la generación de estudios empíricos de genética de comunidades, tomando como marco evolutivo la interacción entre las comunidades de especies arbóreas y polinizadores nativos. Para ello se recopilan interacciones reportadas entre algunos insectos polinizadores y especies leñosas nativas, las que es necesario profundizar con nuevos antecedentes y validar a través de la investigación multidisciplinaria. Conjuntamente, se recopilan los estudios de interacciones genéticas en estas especies, de modo de en el corto plazo poder priorizar aquellas interacciones genéticas y ecológicas que permitan la conservación y evolución de los ecosistemas, bajo un escenario de cambio climático fluctuante.

Se enfatiza que este trabajo es preliminar y no tiene como objetivo compilar para discutir las interacciones ecológicas y genéticas polinizador-planta, dado que la información aún es escasa, pero si se espera incentivar más investigación en el tema, que sin duda es relevante dado el rol vital de los polinizadores en la preservación de los ecosistemas.

\section{OBJETIVO}

Aportar antecedentes preliminares de las interacciones ecológicas y antecedentes genéticos de las especies arbóreas nativas y polinizadores para su aplicación en estudios empíricos de genética de comunidades.

\section{MATERIALES Y MÉTODOS}

Se confeccionó una base de datos donde se confrontan especies leñosas nativas, en relación a insectos polinizadores nativos reportados en artículos científicos, guías de campo e informes técnicos, entre otros. Adicionalmente se incluyó la presencia de polen de especies nativas en la producción de alrededor de 120 muestras de miel de las regiones de Bio Bio y Ñuble, tipificadas a través de ensayos melisopalinológicos durante los años 2017 a 2019. Con la información recopilada se determinó la frecuencia absoluta de interacciones entre planta - polinizador, en especies leñosas.

Se realizó una búsqueda de artículos publicados que cuantifican los efectos de la diversidad intraespecífica en especies leñosas nativas, sobre la estructura de la comunidad y/o el funcionamiento del ecosistema, centrándose en el servicio de polinización. La búsqueda se centró en estudios experimentales que manipulan la variación y/o riqueza intraespecífica dentro de una sola especie para probar sus respectivos efectos ecológicos.

Los estudios que varían la diversidad intraespecífica dentro de un conjunto de especies múltiples no se incluyeron en el meta análisis. También se analizó la lista de referencias de cada artículo para obtener artículos adicionales.

Las siguientes palabras claves (en inglés) se utilizaron en varias combinaciones: "pollinator + community genetics", "pollinator intraspecific variation", "Chilean native pollinators", "Pollination in Chilean forest ecosystems", "Pollinator plant structure", "Pollinator plant interaction".

\section{RESULTADOS}

\section{Interacciones Ecológicas}

La generación de estudios empíricos que permitan estudiar la genética de comunidades necesita contar con una serie de recursos y debe tomar como marco evolutivo las interacciones ecológicas que ocurren dentro de un ecosistema.

En Chile, existen escasos reportes que recopilan interacciones planta -polinizador, se destacan los trabajos de la Dra. Cecilia Smith-Ramírez, afiliada al Instituto de Ecología y Biodiversidad (IEB), Facultad de Ciencias, Universidad de Chile (Smith-Ramírez et al., 2005; Smith-Ramírez y Yáñez, 2010) y el Dr. Víctor Monzon Godoy, académico de la Universidad Católica del Maule (Monzón, 2015), y el reciente 
proyecto "Protegiendo la Biodiversidad y Múltiples Servicios Ecosistémicos en Corredores Biológicos de Montaña, del Ecosistema Mediterráneo de Chile", denominado de modo más breve "GEF Montaña", desarrollado por el Ministerio del Medio Ambiente y la agencia ONU Medio Ambiente, y financiada por el Fondo Mundial para el Medio Ambiente, el cual ha publicado la Guía Insectos Polinizadores Nativos de la Zona Central de Chile (https://gefmontana.mma.gob.cl/categoria/estudios/page/2/).

Para especies forestales, estos antecedentes son aún menores, tal y como ocurre en otras disciplinas, la mayoría de estudios sobre polinizadores están sesgados hacia las áreas templadas y llevados a cabo en países desarrollados, por lo cual todavía existe un gran vacío en Latinoamérica y el Caribe, región en que los países dependen económicamente de la producción agrícola y forestal, y consecuentemente de la polinización.

En la Figura 1 se muestra la frecuencia absoluta de insectos polinizadores reportados en especies nativas arbóreas. Se encontraron 257 interacciones en la bibliografía consultada, el orden predominante de polinizadores corresponde a Himenóptera (51\%), Díptera (32\%), Coleóptera (16\%) y Lepidóptera $(1 \%)$. Esto ya había sido reportado tempranamente por algunos autores que investigaron los ecosistemas de zona andina de Chile central (Arroyo et al., 1982).

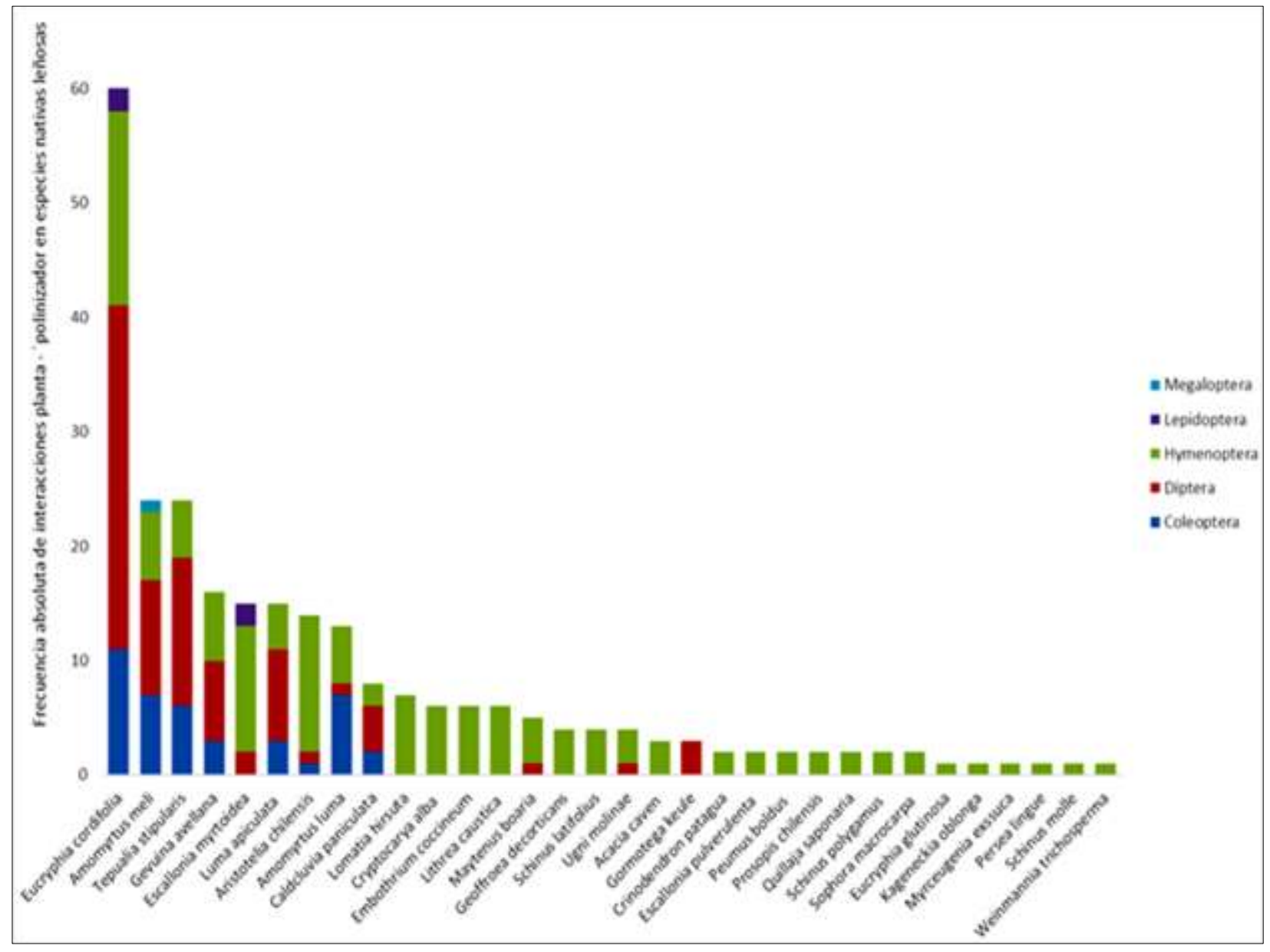

Figura 1. Frecuencia Absoluta de Interacciones Reportadas entre Insectos Polinizadores y Especies Arbóreas Nativas

La especie nativa arbórea que reporta la mayor cantidad de interacciones reportadas corresponde Eucryphia cordifolia (ulmo), reportándose 60 interacciones, esto debido al gran valor melífero que posee la especie, por la abundancia y alta calidad de su néctar, y sus propiedades funcionales demostradas (Montenegro y Ortega, 2013). 
Las especies leñosas clasificadas con algún grado de amenaza según el reglamento de Clasificación de Especies del Ministerio del Medioambiente (RCE - MMA) Gormotega keule y Prosopis chilensis, presentan 3 y 2 interacciones con polinizadores nativos, respectivamente, mientras que Eucryphia glutinosa solo presenta interacción con la especie exótica Apis mellifera (abeja) (Figura 2), que es una de las especies de polinizadores más reconocida en el mundo.

No obstante, a pesar de que $A$. mellifera es ampliamente usada en la producción de miel, algunos autores consideran que no es el mejor polinizador para su uso en algunos cultivos, debido al polimorfismo de las estructuras florales, requiriéndose de otras especies polinizadoras, incluyendo las especies nativas. Tal como es el caso de la especie Persea americana (palto), donde se ha reportado la interacción con varios polinizadores nativos tales como Acamptopoeum submetallicum, Allograpta pulchra, Caenohalictus monilicornis, Corynura chilensis, Corynura chloris, Eristalis tenax, Mordella luctuosa, Polistes buyssoni y Syrphus octomaculatus (Smith-Ramírez y Yáñez, 2010).
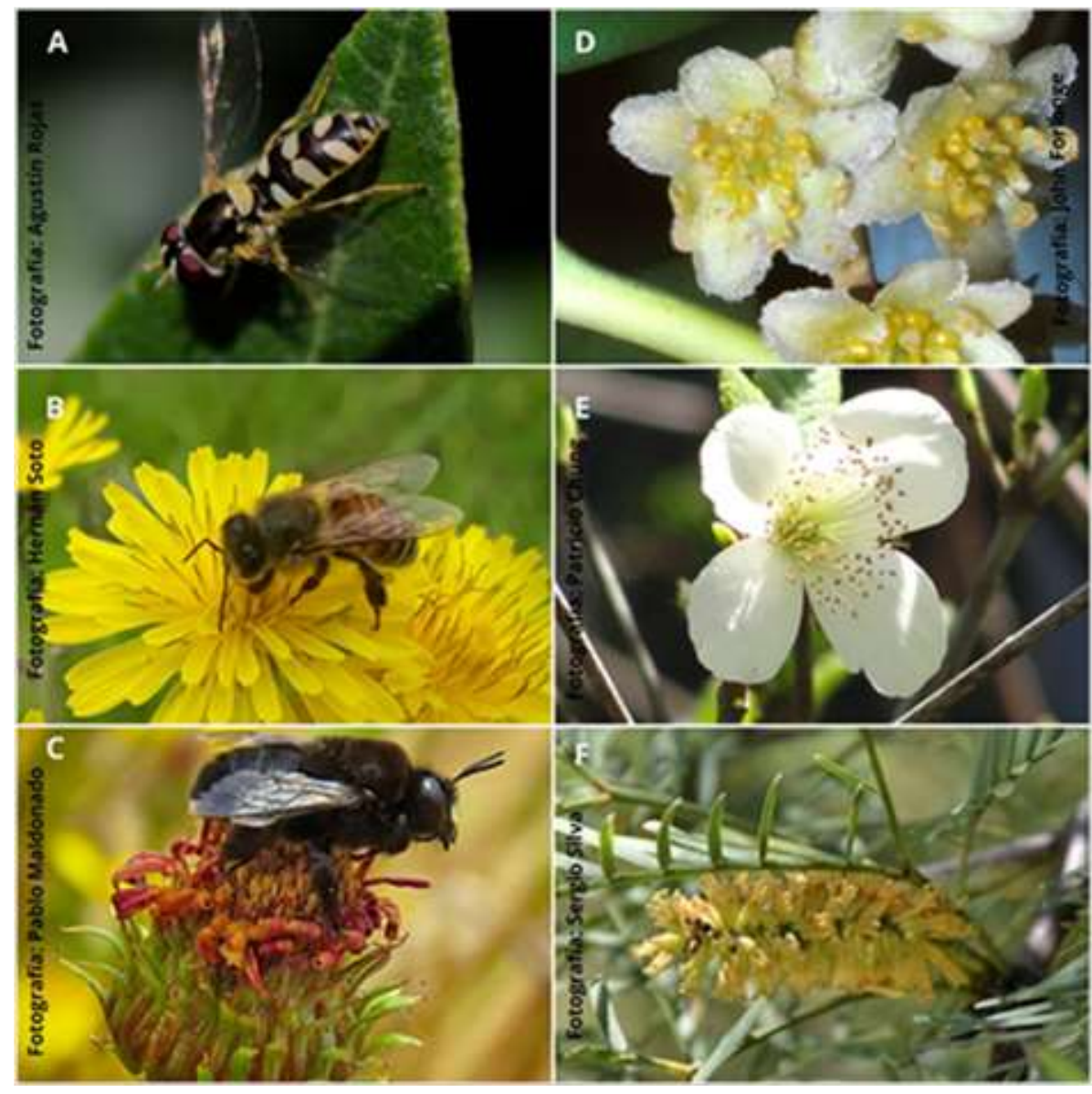

(A) Allograpta hortensis - (D) Gormotega keule (Clasificación: EN); (B) Apis mellifera - (E) Eucryphia glutinosa (Clasificación: VU); (C) Centris nigérrima - (F) Prosopis chilensis (Clasificación: VU).

Figura 2. Interacciones Planta - Polinizador en Especies Leñosas Clasificadas con Grado de Amenaza según el Reglamento de Clasificación de Especies del Ministerio del Medioambiente

Algunas especies siempreverdes con interacciones reportadas; Amomyrtus meli (meli) y Tepualia stipularis (tepú) con 24 interacciones, Gevuina avellana (avellano), Luma apiculata (arrayán)y Aristotelia chilensis (maqui) con 16, 15 y 14 interacciones, respectivamente, y la especie esclerófila Escallonia myrtoidea (lun), con 15 interacciones. 
Las demás especies consultadas muestran entre 1 y 8 interacciones, siendo su moda 2 (Figura 2 y Anexo).

En relación con los polinizadores nativos, la especie que reporta la mayor cantidad de interacciones es Bombus dahlbomii con 12, mientras que las especies Corynura chloris, Cadeguala occidentalis y Allograpta pulchra reportan 10, 7 y 6 , respectivamente.

Los demás polinizadores presentan entre 1 a 5 interacciones, siendo su moda 1. Lo que no significa que se trate de relaciones especifica propiamente, sino más bien se ilustra la falta de estudios que profundicen las interacciones entre los polinizadores nativos.

\section{Interacciones Genéticas}

Un rasgo importante que define el éxito reproductivo de las plantas es el tamaño de la flor o inflorescencia. Entre las consecuencias descritas como favorables al aumento de la floración se encuentran:

- Competencia inter e intraespecífica por los polinizadores.

- Potencial para una mayor producción de semillas.

- Atracción de dispersores de semillas.

- Saturación de depredadores que se alimentan de semillas.

Sin embargo, una producción masiva de flores puede restringir el movimiento de polinizadores entre diferentes individuos (Snow et al., 1996) y la autopolinización puede reemplazar la polinización cruzada y conducir a una mayor endogamia (Valdivia y Niemeyer, 2006).

Otro rasgo recientemente estudiado apunta a que las plantas pueden aumentar su atractivo para los polinizadores aumentando la producción de aromas florales, los cuales se expresan como compuestos orgánicos volátiles (Ramya et al., 2020; Vannette, 2020). De hecho, numerosas investigaciones de laboratorios etológico indican la importancia de los aromas en el comportamiento de los polinizadores y su probable efecto en rendimiento de la planta (Genung et al., 2010). Por lo tanto, no es de extrañar que la variación intraespecífica de los aromas florales influya en la frecuencia de visitas de los polinizadores en las poblaciones naturales

En Chile, para especies forestales estos tipos de estudios son escasos, solo existe un artículo en la bibliografía consultada cuyo objetivo fue describir la interacción de la exhibición floral y la producción de aromas en el éxito reproductivo de Escallonia myrtoidea (Escalloniaceae) en condiciones de campo (Valdivia y Niemeyer, 2006).

Estos autores, después de experimentar manipulaciones del tamaño de la inflorescencia y el olor no observaron efectos no aditivos significativos mediados por los polinizadores en el éxito reproductivo femenino de E. myrtoidea. Concluyendo que la selección de rasgos florales depende de las variaciones en la abundancia de polinizadores a escalas temporal y espacial, lo que dificulta la evaluación de la selección en los rasgos reproductivos dentro de una sola temporada. Desde el punto de vista evolutivo, los autores concluyen que son las diferencias en el éxito reproductivo entre individuos (genotipos), más que entre tipos de inflorescencias, lo que es importante. Por lo que los resultados basados en comparaciones entre inflorescencias, deben ser corroborados en un contexto entre genotipos antes de poder ofrecer interpretaciones evolutivas rigurosas.

Desde la perspectiva de la ciencia de los ecosistemas, la búsqueda de interacciones genéticas a escalas superiores es avance especialmente importante, ya que este campo no ha incorporado previamente la genética en estudios de procesos fundamentales, como el flujo de energía o el ciclo de nutrientes (Whitham et al., 2006; Casacuberta et al., 2016). El examen del papel de las interacciones genéticas a nivel de ecosistema inicia una nueva era de evaluación de ecosistemas dentro de un marco evolutivo.

\section{CONCLUSIONES Y RECOMENDACIONES}

La conservación de los ecosistemas forestales, incluyendo la comunidad asociada, es fundamental para sostener los productos y servicios ecosistémicos que los bosques proveen a la sociedad. Para lograr un avance significativo esta tarea no debe estar al margen de la integración de diversas teorías y conceptos, 
junto con el uso de modernas herramientas empleadas en la genética de comunidades, a fin de obtener mayor poder predictivo de la evolución de las especies, ante un escenario climático fluctuante.

Es un hecho que la disminución de la diversidad vegetal altera las redes ecológicas, como las interacciones planta-polinizador, entre otras. Sin embargo, el conocimiento de los posibles mecanismos que subyacen a los efectos de la pérdida de especies de plantas en estas interacciones es aún limitado y no es posible cuantificar.

Es por esto que se requiere de estudios multidisciplinarios que avancen en la identificación de interacciones ecológicas, y de pruebas experimentales para evaluar los aspectos genéticos que subyacen en estas interacciones.

\section{REFERENCIAS}

Antonovic, J. (1992). Toward community genetics. En: Fritz, R., Simms, E. (Eds). Plant resistance to herbivores and pathogens:ecology, evolution and genetics. University of Chicago Press, Chicago, Illinois, USA. Pp: 426-429.

Arroyo, M., Primack, R. \& Armesto, J. (1982). Community studies in pollination ecology in the high temperate Andes of central Chile. I. Pollination mechanisms and altitudinal variation. American Journal of Botany, 69(1): 82-97. https://doi.org/10.1002/j.15372197.1982.tb13237.x

Benfey, P. \& Mitchell-Olds, T. (2008). From genotype to phenotype: systems biology meets natural variation. Science, 320(5875): 495-497. https://doi.org/10.1126/science.1153716.

Casacuberta, J., Jackson, S., Panaud, O., Purugganan, M. \& Wendel, J. (2016). Evolution of plant phenotypes, from genomes to traits. Genes, Genomes, Genetics, 6(4): 775-778. https://doi.org/10.1534/g3.115.025502.

Donoso, C. \& Gallo, L. (2004). Aspectos conceptuales y metodológicos. En: Donoso, C., Premoli, A., Gallo, L. \& Ipinza, R. Variación intraespecífica en las especies arbóreas de los bosques templados de Chile y Argentina. Editorial Universitaria. Pp: 23-37.

FAO-PNUMA. (2020). El estado de los bosques del mundo 2020. Los bosques, la biodiversidad y las personas. Roma.

Fundación Abejas de Chile. (2020). https://abejasdechile.com/galeria/. Consulta: 29 diciembre, 2020.

Genung, M., Lessard, J., Brown, C., Bunn, W., Cregger, M., Reynolds, W. et al. (2010). Non-Additive Effects of Genotypic Diversity Increase Floral Abundance and Abundance of Floral Visitors. PLoS ONE 5(1): e8711. https://doi.org/10.1371/journal.pone.0008711.

INFOR, 2020. Datos colectados en el Programa FNDR Transferencia Flora Forestal Melífera para Mejorar el Negocio Apícola del Biobío, Fase II 2018 - 2022. Instituto Forestal (datos no publicados).

Jump, A., Marchant, R. \& Penuelas, J. (2009). Environmental change and the option value of genetic diversity. Trends Plant Sciences, 14. Pp: 51-58. https://doi.org/10.1016/i.tplants.2008.10.002.

Medel, R., González-Browne. C. \& Fontúrbel, F.E. (2017). Pollination in the Chilean Mediterranean-Type Ecosystem: a review of current advances and pending tasks. Plant Biology, 20. Pp: 89-99. https://doi.org/10.1111/plb.12644.

Mendoza, J. (2012). Entomofauna asociada a flores de notro (Embothrium coccineum J.R. et G. Forster) y maqui (Aristotelia chilensis (Mol.) Stuntz) en la zona de Valdivia. Tesis presentada como parte de los requisitos para optar al grado de Licenciado en Agronomía. Facultad de Ciencias Agrarias. Universidad Austral de Chile.

MMA-ONU Medio Ambiente. (2020). Guía de Bolsillo: Insectos Polinizadores Nativos de la Zona Central de Chile. Especialistas coautores: Víctor Monzón, Luisa Ruz, Rodrigo Barahona, Vanessa Durán, Cristian Villagra, Patricia Henríquez-Piskulich y Patricia Estrada. Desarrollado y financiado en el marco del Proyecto GEFSEC ID 5135 Ministerio del Medio Ambiente - ONU Medio Ambiente. Santiago, Chile. 68 p.

Montenegro, G. \& Ortega, X. (2013). Innovación y valor agregado en los productos apícolas. Diferenciación y nuevos usos industriales. Apicultura. Informe de Experto. Agrimundo. ODEPA- FIA.

Monzón, V. (2015). Guía de abejas nativas de la Región del Maule-Chile. Universidad Católica del Maule.

Ramya, M., Jang, S., An, H., Lee, S., Park, P. M. \& Park, P. (2020). Volatile organic compounds from orchids: From synthesis and function to gene regulation. International Journal of Molecular Sciences, 21(3): 1160. https://doi.org/10.3390/ijms21031160.

Smith-Ramírez, C., Martínez, P., Nuñez, M., González, C. \& Armesto, J. (2005). Diversity, flower visitation frequency and generalism of pollinators in temperate rain forests of Chiloé Island, Chile. Botanical Journal of the Linnean Society, 147. Pp: $399-416$. https://doi.org/10.1111/j.1095-8339.2005.00388.x.

Smith-Ramírez, C. \& Yáñez, K. (2010). Digitalización de datos de polinizadores de chile, interacción insecto-planta y distribución de insectos. Informe Técnico Final, Red Iberoamericana de Información sobre la Biodiversidad. 
Snow, A., Spira, T., Simpson, R. \& Klips, R. (1996). The ecology of geitonogamous pollination. En: Lloyd, D., Barrett, S (Eds). 1996. Floral Biology: Studies in Floral Evolution in Animal-Pollinated Plants. Chapman \& Hall, New York. Pp: $191-216$. https://doi.org/10.1007/978-1-4613-1165-2 7.

Stadler, P. \& Stephens, C. (2002). Landscapes and Effective Fitness. Comments? Theoretical Biology, 8. Pp: $389-431$. https://doi.org/10.1080/08948550302439.

Templeton, A. (1994). Biodiversity at the molecular genetic level: experiences from disparate macroorganisms. Philosophical Transactions of the Royal Society of London, 345. Pp: 59-64. https://doi.org/10.1098/rstb.1994.0086.

Valdivia, C. \& Niemeyer, H. (2006). ¿Do pollinators simultaneously select for inflorescence size and amount of floral scents? An experimental assessment on Escallonia myrtoidea. Austral Ecology, 31(7): 897-903. https://doi.org/10.1111/j.14429993.2006.01662.x.

Vannette, R. (2020). The floral microbiome: Plant, pollinator, and microbial perspectives. Annual Review of Ecology, Evolution, and Systematics, 51. Pp: 363-386. https://doi.org/10.1146/annurev-ecolsys-011720-013401.

Whitham, T., Young, W., Martinsen, G., Gehring, C., Schweitzer, J., Shuster, S. et al. (2003). Community and ecosystem genetics: A consequence of the extended phenotype. Ecology, 84. Pp: 559-573. https://doi.org/10.1890/00129658(2003)084[0559:CAEGAC]2.0.CO;2

Whitham, T., Bailey, J., Schweitzer, J., Shuster, S., Bangert, R., Le Roy, C. et al. (2006). A framework for community and ecosystem genetics from genes to ecosystems. Nature Reviews: Genetics, 7. Pp: 510-523. https://doi.org/10.1038/nrg1877.

Whitham, T., Allan, G., Cooper, H. \& Shuster, S. (2020). Intraspecific Genetic Variation and Species Interactions Contribute to Community Evolution. Annual Review of Ecology, Evolution, and Systematics, 51. Pp: $587-612$. https://doi.org/10.1146/annurev-ecolsys-011720-123655. 
ANEXO

Lista de Interacciones Planta - Polinizador en Especies Leñosas Nativas

\begin{tabular}{|c|c|c|c|}
\hline PLANTA & POLINIZADOR & ORDEN & REFERENCIA \\
\hline \multirow{3}{*}{ Acacia caven } & Alloscirtetica rufitarsis & $\mathrm{H}$ & Smith - Ramírez y Yáñez, 2010; MMA-ONU, 2020 \\
\hline & Alloscirtetica valparadisaea & $\mathrm{H}$ & Smith - Ramírez y Yáñez, 2010 \\
\hline & Apis mellifera * & $\mathrm{H}$ & INFOR, 2020 \\
\hline \multirow{13}{*}{ Amomyrtus luma } & Apis mellifera * & $\mathrm{H}$ & INFOR, 2020 \\
\hline & Clamirius apicarius & C & Smith - Ramírez et al., 2005 \\
\hline & Dasydema hirtella & C & Smith - Ramírez et al., 2005 \\
\hline & Eurymetopun prasinum & $\mathrm{C}$ & Smith - Ramírez et al., 2005 \\
\hline & Eurymetopun proteus & $\mathrm{C}$ & Smith - Ramírez et al., 2005 \\
\hline & Hylodanacaea elegans & $\mathrm{C}$ & Smith - Ramírez et al., 2005 \\
\hline & Schizochelus serratus & C & Smith - Ramírez et al., 2005 \\
\hline & Stenorhopalus gracilis & $\mathrm{C}$ & Smith - Ramírez et al., 2005 \\
\hline & Melanostoma sp & $\mathrm{D}$ & Smith - Ramírez et al., 2005 \\
\hline & Bombus dahlbomii & $\mathrm{H}$ & $\begin{array}{l}\text { Smith - Ramírez et al., 2005; Smith - Ramírez y Yáñez } \\
\text { 2010; MMA-ONU } 2020\end{array}$ \\
\hline & Cadeguala albopilosa & $\mathrm{H}$ & Smith - Ramírez et al., 2005 \\
\hline & Cadeguala occidentalis & $\mathrm{H}$ & Smith - Ramírez et al., 2005 \\
\hline & Corynura patagónica & $\mathrm{H}$ & $\begin{array}{l}\text { Smith - Ramírez et al., 2005; Smith - Ramírez y Yáñez } \\
2010\end{array}$ \\
\hline \multirow{10}{*}{ Amomyrtus meli } & Adalia deficiens & $\mathrm{C}$ & Smith - Ramírez et al., 2005 \\
\hline & Apis mellifera & $\mathrm{H}$ & $\begin{array}{l}\text { Smith - Ramírez et al., 2005; Smith - Ramírez y Yáñez } \\
2010\end{array}$ \\
\hline & Callideriphus laetus & C & Smith - Ramírez et al., 2005 \\
\hline & Eurymetopun obscurum & $\mathrm{C}$ & Smith - Ramírez et al., 2005 \\
\hline & Hylodanacea binotus & C & Smith - Ramírez et al., 2005 \\
\hline & Hyponotum kraussei & $\mathrm{C}$ & Smith - Ramírez et al., 2005 \\
\hline & Mordella erythrura & C & Smith - Ramírez et al., 2005 \\
\hline & Rhopalomerus tenuirostris & $\mathrm{C}$ & Smith - Ramírez et al., 2005 \\
\hline & Allograpta hortensis & $\mathrm{D}$ & Smith - Ramírez et al., 2005 \\
\hline & Allograpta pulchra & $\mathrm{D}$ & MMA-ONU Medio Ambiente, 2020. \\
\hline
\end{tabular}


Ciencia \& Investigación Forestal Vol. 27 N¹. Abril 2021.

\begin{tabular}{|c|c|c|c|}
\hline PLANTA & POLINIZADOR & ORDEN & REFERENCIA \\
\hline & Cheilosia nitescens & $\mathrm{D}$ & Smith - Ramírez et al., 2005 \\
\hline & Craspedochaeta linbinervis & D & Smith - Ramírez et al., 2005 \\
\hline & Fannia sp & D & Smith - Ramírez et al., 2005 \\
\hline & Melanostoma fenestratus & $\mathrm{D}$ & Smith - Ramírez et al., 2005 \\
\hline & Melanostoma lundbladi & D & Smith - Ramírez et al., 2005 \\
\hline & Melanostoma sp & D & Smith - Ramírez et al., 2005 \\
\hline & Mesograpta philippi & $\mathrm{D}$ & Smith - Ramírez et al., 2005 \\
\hline & Stilbosona cyanea & D & Smith - Ramírez et al., 2005 \\
\hline & Bombus dahlbomii & $\mathrm{H}$ & $\begin{array}{l}\text { Smith - Ramírez et al., 2005; Smith - Ramírez y Yáñez } \\
2010\end{array}$ \\
\hline & Cadeguala albopilosa & $\mathrm{H}$ & Smith - Ramírez et al., 2005 \\
\hline & Corynura corynogastra & $\mathrm{H}$ & $\begin{array}{l}\text { Smith - Ramírez et al., 2005; Smith - Ramírez y Yáñez } \\
2010\end{array}$ \\
\hline & Corynura patagónica & $\mathrm{H}$ & $\begin{array}{l}\text { Smith - Ramírez et al., 2005; Smith - Ramírez y Yáñez } \\
2010\end{array}$ \\
\hline & Diphaglossa gayi & $\mathrm{H}$ & $\begin{array}{l}\text { Smith - Ramírez et al., 2005; Smith - Ramírez y Yáñez } \\
2010\end{array}$ \\
\hline & Protosialis chilensis & M & Smith - Ramírez et al., 2005 \\
\hline \multirow{13}{*}{ Aristotelia chilensis } & Apis mellifera & $\mathrm{H}$ & INFOR 2020 \\
\hline & Astylus trifasciatus & C & MMA-ONU 2020 \\
\hline & Carposcalis fenestratum & D & Mendoza 2012 \\
\hline & Bombus dahlbomii & $\mathrm{H}$ & Mendoza 2012 \\
\hline & Cadeguala occidentalis & $\mathrm{H}$ & Mendoza 2012 \\
\hline & Corynura chloris & $\mathrm{H}$ & MMA-ONU 2020 \\
\hline & Caupolicana gayi & $\mathrm{H}$ & MMA-ONU 2020 \\
\hline & Centris cyanescens & $\mathrm{H}$ & MMA-ONU 2020 \\
\hline & Colletes seminitidus & $\mathrm{H}$ & Smith - Ramírez y Yáñez 2010 \\
\hline & Diphaglossa gayi & $\mathrm{H}$ & Mendoza 2012 \\
\hline & Policana albopilosa & $\mathrm{H}$ & Mendoza 2012 \\
\hline & Ruizantheda mutabilis & $\mathrm{H}$ & Mendoza 2012 \\
\hline & Ruizantheda sp & $\mathrm{H}$ & Mendoza 2012 \\
\hline
\end{tabular}


Ciencia \& Investigación Forestal Vol. 27 N¹. Abril 2021.

\begin{tabular}{|c|c|c|c|}
\hline PLANTA & POLINIZADOR & ORDEN & REFERENCIA \\
\hline \multirow{8}{*}{ Caldcluvia paniculata } & Aleocharinae $s p$ & C & Smith - Ramírez et al., 2005 \\
\hline & Apis mellifera * & $\mathrm{H}$ & INFOR 2020 \\
\hline & Mordella erythrura & C & Smith - Ramírez et al., 2005 \\
\hline & Melanostoma chalconotus & D & Smith - Ramírez et al., 2005 \\
\hline & Melanostoma sp & $\mathrm{D}$ & Smith - Ramírez et al., 2005 \\
\hline & Morphodexia sp & $\mathrm{D}$ & Smith - Ramírez et al., 2005 \\
\hline & Peleteria filipalpis & $\mathrm{D}$ & Smith - Ramírez et al., 2005 \\
\hline & Bombus dahlbomii & $\mathrm{H}$ & $\begin{array}{l}\text { Smith - Ramírez et al., 2005; Smith - Ramírez y Yáñez } \\
\text { 2010; MMA-ONU Medio Ambiente, } 2020 .\end{array}$ \\
\hline \multirow[b]{2}{*}{ Crinodendron patagua } & Apis mellifera * & $\mathrm{H}$ & INFOR 2020 \\
\hline & Bombus dahlbomii & $\mathrm{H}$ & Smith - Ramírez y Yáñez 2010; MMA-ONU 2020 \\
\hline \multirow{6}{*}{ Cryptocarya alba } & Apis mellifera * & $\mathrm{H}$ & INFOR 2020 \\
\hline & Corynura chloris & $\mathrm{H}$ & MMA-ONU 2020 \\
\hline & Centris cyanescens & $\mathrm{H}$ & MMA-ONU 2020 \\
\hline & Colletes cyanescens & $\mathrm{H}$ & Mendoza 2012 \\
\hline & Corynura chloris & $\mathrm{H}$ & Mendoza 2012 \\
\hline & Svastrides melanura & $\mathrm{H}$ & Mendoza 2012 \\
\hline \multirow{5}{*}{ Embothrium coccineum } & Apis mellifera * & $\mathrm{H}$ & $\begin{array}{l}\text { Smith - Ramírez et al., 2005; Smith - Ramírez y Yáñez } \\
\text { 2010; INFOR. } 2020\end{array}$ \\
\hline & Cadeguala occidentalis & $\mathrm{H}$ & Mendoza 2012 \\
\hline & Diphaglossa gayi & $\mathrm{H}$ & $\begin{array}{l}\text { Smith - Ramírez et al., 2005; Smith - Ramírez y Yáñez } \\
2010\end{array}$ \\
\hline & Ruizantheda mutabilis & $\mathrm{H}$ & Mendoza 2012 \\
\hline & Ruizantheda sp & $\mathrm{H}$ & Mendoza 2012 \\
\hline \multirow{6}{*}{ Escallonia myrtoidea } & Apis mellifera * & $\mathrm{H}$ & Smith - Ramírez y Yáñez 2010 \\
\hline & Copestylum bradleyi & $\mathrm{D}$ & Smith - Ramírez y Yáñez 2010 \\
\hline & Dasybasis pruinivitta & $\mathrm{D}$ & Smith - Ramírez y Yáñez 2010 \\
\hline & Bombus dahlbomii & $\mathrm{H}$ & Smith - Ramírez y Yáñez 2010; MMA-ONU 2020 \\
\hline & Colletes murinus & $\mathrm{H}$ & Smith - Ramírez y Yáñez 2010 \\
\hline & Corynura chilensis & $\mathrm{H}$ & Smith - Ramírez y Yáñez 2010 \\
\hline
\end{tabular}


Ciencia \& Investigación Forestal Vol. 27 N¹. Abril 2021.

\begin{tabular}{|c|c|c|c|}
\hline PLANTA & POLINIZADOR & ORDEN & REFERENCIA \\
\hline & Corynura lepida & $\mathrm{H}$ & Smith - Ramírez y Yáñez 2010 \\
\hline & Hypodinerus colocolo & $\mathrm{H}$ & Smith - Ramírez y Yáñez 2010 \\
\hline & Pepsis limbata & $\mathrm{H}$ & Smith - Ramírez y Yáñez 2010 \\
\hline & Polistes buyssoni & $\mathrm{H}$ & Smith - Ramírez y Yáñez 2010 \\
\hline & Ruizantheda proxima & $\mathrm{H}$ & Smith - Ramírez y Yáñez 2010 \\
\hline & Sphecodes chilensis & $\mathrm{H}$ & Smith - Ramírez y Yáñez 2010 \\
\hline & Stenodynerus scabriusculus & $\mathrm{H}$ & Smith - Ramírez y Yáñez 2010 \\
\hline & Pseudolucia chilensis & L & Smith - Ramírez y Yáñez 2010 \\
\hline & Vannesa carye & $\mathrm{L}$ & Smith - Ramírez y Yáñez 2010; MMA-ONU 2020 \\
\hline \multirow[b]{2}{*}{ Escallonia pulverulenta } & Apis mellifera & $\mathrm{H}$ & INFOR, 2020 \\
\hline & Lonchopria zonalis & $\mathrm{H}$ & Fundación Abejas de Chile, 2020. \\
\hline \multirow{16}{*}{ Eucryphia cordifolia } & Apis mellifera & $\mathrm{H}$ & $\begin{array}{l}\text { Smith - Ramírez et al., 2005; Smith - Ramírez y Yáñez } \\
\text { 2010; INFOR, } 2020\end{array}$ \\
\hline & Bilyaxia concinna & $\mathrm{C}$ & Smith - Ramírez y Yáñez, 2010 \\
\hline & Callideriphus laetus & C & Smith - Ramírez y Yáñez 2010 \\
\hline & Chauliognathus sp & C & Smith - Ramírez et al., 2005 \\
\hline & Hyponotum kraussei & $\mathrm{C}$ & Smith - Ramírez et al., 2005 \\
\hline & Mecopselaphus maculicollis & C & Smith - Ramírez et al., 2005 \\
\hline & $\begin{array}{l}\text { Melanophthalma aff } \\
\text { seminigra }\end{array}$ & C & Smith - Ramírez y Yáñez, 2010 \\
\hline & Mordella erythrura & C & $\begin{array}{l}\text { Smith - Ramírez et al., 2005; Smith - Ramírez y Yáñez, } \\
2010\end{array}$ \\
\hline & Mordella luctuosa & C & Smith - Ramírez y Yáñez, 2010 \\
\hline & Platinocera gracilis & C & Smith - Ramírez y Yáñez ,2010 \\
\hline & Platynocera gracilipes & C & Smith - Ramírez y Yáñez ,2010 \\
\hline & Rhopalomerus tenuirostris & C & Smith - Ramírez et al., 2005 \\
\hline & Agelanius meridiana & $\mathrm{D}$ & Smith - Ramírez y Yáñez, 2010 \\
\hline & Allograpta hortensis & $\mathrm{D}$ & Smith - Ramírez y Yáñez, 2010 \\
\hline & Allograpta pulchra & $\mathrm{D}$ & Smith - Ramírez y Yáñez, 2010 \\
\hline & Aneriophora aureorrufa & D & Smith - Ramírez y Yáñez,2010 \\
\hline
\end{tabular}




\begin{tabular}{|c|c|c|c|}
\hline PLANTA & POLINIZADOR & ORDEN & REFERENCIA \\
\hline & Cheilosia nitescens & D & Smith - Ramírez y Yáñez, 2010 \\
\hline & Dasyoma coeruleum & $\mathrm{D}$ & Smith - Ramírez y Yáñez,2010 \\
\hline & Dolichogyna chilensis & D & Smith - Ramírez y Yáñez. 2010 \\
\hline & Eristalis assimilis & D & $\begin{array}{l}\text { Smith - Ramírez et al., 2005; Smith - Ramírez y Yáñez, } \\
2010\end{array}$ \\
\hline & Eristalis elegans & D & $\begin{array}{l}\text { Smith - Ramírez et al., 2005; Smith - Ramírez y Yáñez, } \\
2010\end{array}$ \\
\hline & Eristalis meigenii & D & Smith - Ramírez y Yáñez, 2010 \\
\hline & Eristalis tenax & D & Smith - Ramírez y Yáñez, 2010 \\
\hline & Eurygastromyia philippi & D & Smith - Ramírez y Yáñez, 2010 \\
\hline & Fannia $s p$ & D & Smith - Ramírez et al., 2005 \\
\hline & Fazia macquarti & $\mathrm{D}$ & Smith - Ramírez y Yáñez ,2010 \\
\hline & Lypha erigonopsidis & $\mathrm{D}$ & Smith - Ramírez et al., 2005 \\
\hline & Macrometopia atra & D & Smith - Ramírez et al., 2005 \\
\hline & Megalybus crassus & D & Smith - Ramírez et al., 2005 \\
\hline & Melanostoma chalconotus & D & $\begin{array}{l}\text { Smith - Ramírez et al., 2005; Smith - Ramírez y Yáñez, } \\
2010\end{array}$ \\
\hline & Melanostoma fenestratus & D & $\begin{array}{l}\text { Smith - Ramírez et al., 2005; Smith - Ramírez y Yáñez, } \\
2010\end{array}$ \\
\hline & Melanostoma lundbladi & D & Smith - Ramírez y Yáñez, 2010 \\
\hline & Mesograpta calceolatus & D & Smith - Ramírez et al., 2005 \\
\hline & Mesograpta philippi & D & Smith - Ramírez y Yáñez, 2010 \\
\hline & Mycteromia conica & D & Smith - Ramírez y Yáñez, 2010 \\
\hline & Peleteria filipalpis & D & Smith - Ramírez y Yáñez ,2010 \\
\hline & Platycheirus chalconota & D & MMA-ONU, 2020 \\
\hline & Syrphus octomaculatus & D & Smith - Ramírez y Yáñez, 2010 \\
\hline & Trichophthalma barbarrosa & D & Smith - Ramírez y Yáñez , 2010 \\
\hline & Trichophthalma commutata & D & $\begin{array}{l}\text { Smith - Ramírez et al., 2005; Smith - Ramírez y Yáñez, } \\
2010\end{array}$ \\
\hline & Trichophthalma herbsti & D & $\begin{array}{l}\text { Smith - Ramírez et al., 2005; Smith - Ramírez y Yáñez, } \\
2010\end{array}$ \\
\hline & Tropidia sp & D & Smith - Ramírez et al., 2005 \\
\hline
\end{tabular}




\begin{tabular}{|c|c|c|c|}
\hline PLANTA & POLINIZADOR & ORDEN & REFERENCIA \\
\hline & Bombus dahlbomii & $\mathrm{H}$ & $\begin{array}{l}\text { Smith - Ramírez et al., 2005; Smith - Ramírez y Yáñez, } \\
\text { 2010; MMA-ONU, } 2020\end{array}$ \\
\hline & Cadeguala occidentalis & $\mathrm{H}$ & Smith - Ramírez y Yáñez, 2010 \\
\hline & Caenohalictus monilicornis & $\mathrm{H}$ & Smith - Ramírez y Yáñez,2010 \\
\hline & Corynura atrovirens & $\mathrm{H}$ & Smith - Ramírez y Yáñez, 2010 \\
\hline & Corynura chilensis & $\mathrm{H}$ & Smith - Ramírez y Yáñez, 2010 \\
\hline & Corynura chloris & $\mathrm{H}$ & Smith - Ramírez y Yáñez, 2010 \\
\hline & Corynura corinogaster & $\mathrm{H}$ & Smith - Ramírez y Yáñez, 2010 \\
\hline & Corynura heterochlora & $\mathrm{H}$ & Smith - Ramírez y Yáñez, 2010 \\
\hline & Corynura lepida & $\mathrm{H}$ & Smith - Ramírez y Yáñez, 2010 \\
\hline & Corynura patagónica & $\mathrm{H}$ & Smith - Ramírez y Yáñez, 2010 \\
\hline & Corynura rubella & $\mathrm{H}$ & Smith - Ramírez y Yáñez, 2010 \\
\hline & Diphaglossa gayi & $\mathrm{H}$ & Smith - Ramírez y Yáñez, 2010 \\
\hline & Eumeninae $s p$ & $\mathrm{H}$ & Smith - Ramírez et al., 2005 \\
\hline & Hypodynerus spp & $\mathrm{H}$ & Smith - Ramírez et al., 2005 \\
\hline & Ruizantheda mutabilis & $\mathrm{H}$ & Smith - Ramírez y Yáñez 2010 \\
\hline & Ruizantheda proxima & $\mathrm{H}$ & Smith - Ramírez y Yáñez, 2010 \\
\hline & Colias vauthierii & L & Smith - Ramírez y Yáñez, 2010 \\
\hline & Eroessa chiliensis & L & Smith - Ramírez et al., 2005 \\
\hline \multirow{4}{*}{ Geoffroea decorticans } & Alloscirtetica gayi & $\mathrm{H}$ & Smith - Ramírez y Yáñez, 2010 \\
\hline & Alloscirtetica rufitarsis & $\mathrm{H}$ & Smith - Ramírez y Yáñez, 2010; MMA-ONU, 2020 \\
\hline & Alloscirtetica valparadisaea & $\mathrm{H}$ & Smith - Ramírez y Yáñez, 2010 \\
\hline & Centris nigerrima & $\mathrm{H}$ & Smith - Ramírez y Yáñez, 2010 \\
\hline \multirow{6}{*}{ Gevuina avellana } & Apis mellifera & $\mathrm{H}$ & $\begin{array}{l}\text { Smith - Ramírez et al., 2005; Smith - Ramírez y Yáñez, } \\
\text { 2010; INFOR, } 2020\end{array}$ \\
\hline & Aleocharinae $s p$ & C & Smith - Ramírez et al., 2005 \\
\hline & Sapromyza edwardsi & C & Smith - Ramírez y Yáñez, 2010 \\
\hline & Sapromyza micropyga & C & Smith - Ramírez y Yáñez, 2010 \\
\hline & Allograpta hortensis & $\mathrm{D}$ & Smith - Ramírez y Yáñez ,2010; MMA-ONU, 2020 \\
\hline & Allograpta pulchra & $\mathrm{D}$ & Smith - Ramírez et al., 2005; MMA-ONU, 2020 \\
\hline
\end{tabular}


Ciencia \& Investigación Forestal Vol. 27 N¹. Abril 2021.

\begin{tabular}{|c|c|c|c|}
\hline PLANTA & POLINIZADOR & ORDEN & REFERENCIA \\
\hline & Eristalis tenax & $\mathrm{D}$ & Smith - Ramírez et al., 2005 \\
\hline & Fannia sp & D & Smith - Ramírez et al., 2005 \\
\hline & Melanostoma sp & $\mathrm{D}$ & Smith - Ramírez et al., 2005 \\
\hline & Syrphus octomaculatus & D & Smith - Ramírez y Yáñez, 2010 \\
\hline & Syrphus reedi & $\mathrm{D}$ & Smith - Ramírez y Yáñez,2010 \\
\hline & Cadeguala albopilosa & $\mathrm{H}$ & Smith - Ramírez et al., 2005 \\
\hline & Cadeguala occidentalis & $\mathrm{H}$ & Smith - Ramírez et al., 2005 \\
\hline & Caenohalictus monilicornis & $\mathrm{H}$ & $\begin{array}{l}\text { Smith - Ramírez et al., 2005; Smith - Ramírez y Yáñez, } \\
2010\end{array}$ \\
\hline & Corynura atrovirens & $\mathrm{H}$ & Smith - Ramírez et al., 2005 \\
\hline & Vespula germánica & $\mathrm{H}$ & Smith - Ramírez y Yáñez, 2010 \\
\hline Kageneckia oblonga & Apis mellifera * & $\mathrm{H}$ & INFOR, 2020 \\
\hline \multirow{3}{*}{ Gormotega keule } & Allograpta hortensis & $\mathrm{D}$ & Smith - Ramírez y Yáñez, 2010; MMA-ONU, 2020 \\
\hline & Syrphus octomaculatus & D & Smith - Ramírez y Yáñez, 2010 \\
\hline & Syrphus reedi & $\mathrm{D}$ & Smith - Ramírez y Yáñez, 2010 \\
\hline \multirow{6}{*}{ Lithrea caustica } & Apis mellifera * & $\mathrm{H}$ & INFOR, 2020 \\
\hline & Cadeguala occidentalis & $\mathrm{H}$ & Monzon, 2015 \\
\hline & Centris cyanescens & $\mathrm{H}$ & MMA-ONU Medio Ambiente, 2020. \\
\hline & Colletes seminitidus & $\mathrm{H}$ & Monzon 2015 \\
\hline & Corynura chloris & $\mathrm{H}$ & Monzon, 2015 \\
\hline & Manuelia postica & $\mathrm{H}$ & Monzon, 2015 \\
\hline \multirow{8}{*}{ Lomatia hirsuta } & Apis mellifera * & $\mathrm{H}$ & INFOR, 2020 \\
\hline & Bombus dahlbomii & $\mathrm{H}$ & Monzon, 2015 \\
\hline & Cadeguala occidentalis & $\mathrm{H}$ & Monzon, 2015 \\
\hline & Centris cyanescens & $\mathrm{H}$ & MMA-ONU, 2020 \\
\hline & Colletes seminitidus & $\mathrm{H}$ & Fundación Abejas de Chile, 2020. \\
\hline & Colletes seminitidus & $\mathrm{H}$ & Monzon, 2015 \\
\hline & Manuelia postica & $\mathrm{H}$ & Monzon, 2015 \\
\hline & Apis mellifera * & $\mathrm{H}$ & $\begin{array}{l}\text { Smith - Ramírez et al., 2005; Smith - Ramírez y Yáñez, } \\
\text { 2010; INFOR, } 2020\end{array}$ \\
\hline
\end{tabular}


Ciencia \& Investigación Forestal Vol. 27 N¹. Abril 2021.

\begin{tabular}{|c|c|c|c|}
\hline PLANTA & POLINIZADOR & ORDEN & REFERENCIA \\
\hline \multirow[t]{14}{*}{ Luma apiculata } & Chauliognathus sp & C & Smith - Ramírez et al., 2005 \\
\hline & Dasydema hirtella & $\mathrm{C}$ & Smith - Ramírez et al., 2005 \\
\hline & Mordella erythrura & C & Smith - Ramírez et al., 2005 \\
\hline & Allograpta pulchra & $\mathrm{D}$ & Smith - Ramírez et al., 2005; MMA-ONU, 2020 \\
\hline & Eristalis assimilis & $\mathrm{D}$ & Smith - Ramírez et al., 2005 \\
\hline & Fazia bullaephora & $\mathrm{D}$ & Smith - Ramírez et al., 2005 \\
\hline & Fazia macquarti & $\mathrm{D}$ & Smith - Ramírez et al., 2005 \\
\hline & Megalybus crassus & $\mathrm{D}$ & $\begin{array}{l}\text { Smith - Ramírez et al., 2005; Smith - Ramírez y Yáñez, } \\
2010\end{array}$ \\
\hline & Melanostoma sp & $\mathrm{D}$ & Smith - Ramírez et al., 2005 \\
\hline & Stilbosona cyanea & $\mathrm{D}$ & Smith - Ramírez et al., 2005 \\
\hline & Thrypticus sp & $\mathrm{D}$ & Smith - Ramírez et al., 2005 \\
\hline & Bombus dahlbomii & $\mathrm{H}$ & $\begin{array}{l}\text { Smith - Ramírez et al., 2005; Smith - Ramírez y Yáñez, } \\
\text { 2010; MMA-ONU, } 2020\end{array}$ \\
\hline & Corynura patagónica & $\mathrm{H}$ & $\begin{array}{l}\text { Smith - Ramírez et al., 2005; Smith - Ramírez y Yáñez, } \\
2010\end{array}$ \\
\hline & Corynura rubella & $\mathrm{H}$ & $\begin{array}{l}\text { Smith - Ramírez et al., 2005; Smith - Ramírez y Yáñez } \\
2010\end{array}$ \\
\hline Myrceugenia exssuca & Apis mellifera * & $\mathrm{H}$ & INFOR, 2020 \\
\hline \multirow{5}{*}{ Maytenus boaria } & Allograpta pulchra & $\mathrm{D}$ & MMA-ONU, 2020 \\
\hline & Corynura chloris & $\mathrm{H}$ & MMA-ONU, 2020 \\
\hline & Centris cyanescens & $\mathrm{H}$ & MMA-ONU, 2020 \\
\hline & Corynura cristata & $\mathrm{H}$ & MMA-ONU, 2020 \\
\hline & Corynura herbsti & $\mathrm{H}$ & MMA-ONU 2020 \\
\hline Persea lingue & Manuelia gayatina & $\mathrm{H}$ & Smith - Ramírez y Yáñez, 2010 \\
\hline \multirow[t]{2}{*}{ Peumus boldus } & Corynura chloris & $\mathrm{H}$ & MMA-ONU, 2020 \\
\hline & Corynura chloris & $\mathrm{H}$ & Monzon, 2015 \\
\hline \multirow[t]{2}{*}{ Prosopis chilensis } & Alloscirtetica gayi & $\mathrm{H}$ & Smith - Ramírez y Yáñez, 2010 \\
\hline & Centris nigerrima & $\mathrm{H}$ & Smith - Ramírez y Yáñez 2010 \\
\hline \multirow[b]{2}{*}{ Quillaja saponaria } & Apis mellifera * & $\mathrm{H}$ & INFOR, 2020 \\
\hline & Corynura chloris & $\mathrm{H}$ & MMA-ONU, 2020 \\
\hline
\end{tabular}


Ciencia \& Investigación Forestal Vol. 27 N 1. Abril 2021.

\begin{tabular}{|c|c|c|c|}
\hline PLANTA & POLINIZADOR & ORDEN & REFERENCIA \\
\hline \multirow{4}{*}{ Schinus latifolius } & Apis mellifera * & $\mathrm{H}$ & INFOR, 2020 \\
\hline & Corynura chloris & $\mathrm{H}$ & MMA-ONU, 2020 \\
\hline & Centris cyanescens & $\mathrm{H}$ & MMA-ONU, 2020 \\
\hline & Corynura cristata & $\mathrm{H}$ & MMA-ONU, 2020 \\
\hline Schinus molle & Colletes seminitidus & $\mathrm{H}$ & Monzon, 2015 \\
\hline \multirow[b]{2}{*}{ Schinus polygamus } & Apis mellifera * & $\mathrm{H}$ & INFOR, 2020 \\
\hline & $\begin{array}{l}\text { Acamptopoeum } \\
\text { submetallicum }\end{array}$ & $\mathrm{H}$ & MMA-ONU, 2020 \\
\hline \multirow[b]{2}{*}{ Sophora macrocarpa } & Apis mellifera * & $\mathrm{H}$ & INFOR, 2020 (datos no publicados) \\
\hline & Bombus dahlbomii & $\mathrm{H}$ & Monzon, 2015 \\
\hline \multirow{19}{*}{ Tepualia stipularis } & Apis mellifera * & $\mathrm{H}$ & $\begin{array}{l}\text { Smith - Ramírez et al., 2005; Smith - Ramírez y Yáñez, } \\
\text { 2010; INFOR, } 2020\end{array}$ \\
\hline & Aleocharinae $s p$ & C & Smith - Ramírez et al., 2005 \\
\hline & Chauliognathus sp & C & Smith - Ramírez et al., 2005 \\
\hline & Melanophthalma seminigra & C & Smith - Ramírez et al., 2005 \\
\hline & Mordella erythrura & C & Smith - Ramírez et al., 2005 \\
\hline & Mordella luctuosa & C & Smith - Ramírez et al., 2005 \\
\hline & Rhopalomerus tenuirostris & $\mathrm{C}$ & Smith - Ramírez et al., 2005 \\
\hline & Allograpta hortensis & $\mathrm{D}$ & Smith - Ramírez et al., 2005; MMA-ONU, 2020 \\
\hline & Allograpta pulchra & $\mathrm{D}$ & Smith - Ramírez et al., 2005; MMA-ONU, 2020 \\
\hline & Dolichogyna chilensis & $\mathrm{D}$ & Smith - Ramírez et al., 2005 \\
\hline & Eristalis elegans & D & Smith - Ramírez et al., 2005 \\
\hline & Fannia sp & $\mathrm{D}$ & Smith - Ramírez et al., 2005 \\
\hline & Fazia macquarti & $\mathrm{D}$ & Smith - Ramírez et al., 2005 \\
\hline & Melanostoma chalconotus & $\mathrm{D}$ & Smith - Ramírez et al., 2005 \\
\hline & Melanostoma lundbladi & $D$ & Smith - Ramírez et al., 2005 \\
\hline & Mesograpta philippi & $\mathrm{D}$ & Smith - Ramírez et al., 2005 \\
\hline & Stilbosona cyanea & $\mathrm{D}$ & Smith - Ramírez et al., 2005 \\
\hline & Syrphus octomaculatus & $\mathrm{D}$ & Smith - Ramírez et al., 2005 \\
\hline & Tachininae sp & $\mathrm{D}$ & Smith - Ramírez et al., 2005 \\
\hline
\end{tabular}


Ciencia \& Investigación Forestal Vol. 27 N¹. Abril 2021.

\begin{tabular}{|c|c|c|c|}
\hline PLANTA & POLINIZADOR & ORDEN & REFERENCIA \\
\hline & Thrypticus sp & D & Smith - Ramírez et al., 2005 \\
\hline & Bombus dahlbomii & $\mathrm{H}$ & $\begin{array}{l}\text { Smith - Ramírez et al., 2005; Smith - Ramírez y Yáñez, } \\
\text { 2010; MMA-ONU, } 2020\end{array}$ \\
\hline & Cadeguala albopilosa & $\mathrm{H}$ & Smith - Ramírez et al., 2005 \\
\hline & Eumeninae sp & $\mathrm{H}$ & Smith - Ramírez et al., 2005 \\
\hline & Vespula germánica & $\mathrm{H}$ & $\begin{array}{l}\text { Smith - Ramírez et al., 2005; Smith - Ramírez y Yáñez, } \\
2010\end{array}$ \\
\hline \multirow{4}{*}{ Ugni molinae } & Apis mellifera * & $\mathrm{H}$ & $\begin{array}{l}\text { Smith - Ramírez et al., 2005; Smith - Ramírez y Yáñez. } \\
\text { 2010; INFOR, } 2020\end{array}$ \\
\hline & Melanostoma sp & D & Smith - Ramírez et al., 2005 \\
\hline & Bombus dahlbomii & $\mathrm{H}$ & Smith - Ramírez et al., 2005; MMA-ONU 2020 \\
\hline & Diphaglossa gayi & $\mathrm{H}$ & Smith - Ramírez y Yáñez, 2010 \\
\hline $\begin{array}{l}\text { Weinmannia } \\
\text { trichosperma }\end{array}$ & Apis mellifera * & $\mathrm{H}$ & INFOR, 2020 \\
\hline
\end{tabular}

Ordenes: C: Coleóptera, D: Díptera, H: Himenóptera, L: Lepidóptera, M: Megaloptera.

$\left({ }^{\star}\right)$ Especie exótica 